\title{
Logarithmic Differential Operators and Prequantization of Logsymplectic Poisson Structure
}

\author{
Joseph Dongho ${ }^{1}$ \\ ${ }^{1}$ Department of Mathematics and Computer Sciences University of Maroua, Cameroon \\ Correspondence: Joseph Dongho, Department of Mathematics and Computer Sciences University of Maroua, Cameroon. \\ E-mail: joseph.dongho@fs.univ-maroua.cm
}

Received: June 28, 2017 Accepted: July 13, 2017 Online Published: July 25, 2017

doi:10.5539/jmr.v9n4p231～URL: https://doi.org/10.5539/jmr.v9n4p231

\begin{abstract}
In this paper, we study the prequantization condition of logsymplectic structure using integrality of such structure on the complement of associated divisor $D$.
\end{abstract}

Keywords: Log-symplectic structure, Poisson cohomology, Log-Poisson cohomology, Poisson structures.

\section{Introduction}

The first problem of geometric quantization concerns the kinematic relationship between the classical and quantum domains. At the quantum level, the states of physical system are represented by the rays in Hilbert space $\mathcal{H}$, and the observable by a collection $O$ of symmetric operators on $\mathcal{H}$, while in the limiting classical description, the state space is a symplectic manifold or more generally Poisson manifold $(X, \omega)$ and the observable are algebra $C^{\infty}(X)$ of smooth functions on $X$. The kinematic problem is: given $X$ and $\omega$, is it possible to reconstruct $\mathcal{H}$ and $O$ ? According to Dirac's general principles, the canonical transformations of $X$ generated by the classical observable should correspond to the unitary transformations of $\mathcal{H}$ generated by the quantum observable, and Poisson brackets of classical observable should correspond to commutators of quantum observable. Each classical observable $f: X \rightarrow$ R should correspond to an operator $\varphi(f) \in O$ such that

1. the map $f \rightarrow \varphi(f)$ is linear over $\mathrm{R}$

2. if $f$ is constant, then $\varphi(f)$ is the corresponding multiplication operator

3. $\left[f_{1}, f_{2}\right]=f_{3}$ then $\varphi\left(f_{1}\right) \varphi\left(f_{2}\right)-\varphi\left(f_{2}\right) \varphi\left(f_{1}\right)=-i h \varphi\left(f_{3}\right)$.

Therefore, the Poisson bracket is the classical analogue of the quantum commutator. Linear map $\varphi$ satisfying (1 $\rightarrow 2)$ is called prequantization formula. When the prequantization formula exists, $C^{\infty}(X)$ acts faithfully on the Hilbert space $\mathcal{H}$. In the case where classical phase space if represent by symplectic manifold, J. Soureau and B. Kostant; independently presented necessary and sufficient conditions on which symplectic manifold $(X, \omega)$ shall be prequantizable. Their proved that $(X, \omega)$ is prequantizable if and only if the De Rham cohomolgy class of $\omega$ is element of the image of homology map induced by the inclusion of $\mathbb{Z}$ in $\mathbb{R}$. More generally, I. Vaisman introduced the notion of contravariant derivative, Poisson Chern class and used it to generalize the Kostant-Soureau integral theorem. They prove that the obstruction to prequantization of symplectic manifold is measured by De Rham cohomology while Poisson cohomolgy measures the obstruction to the prequantization of Poisson manifold.

Between symplectic manifold and Poisson manifold, there exist the logarithmic manifold. The notion of logsymplectic structure taking their origin from particular meromorphic forms having at most simple poles along certain divisor $D$ of a giving complex manifold $X$. Such forms are amply studied in (Saito, K. (1980)). Using the notion Lie-Rinehart algebra, we give the algebraic generalization of such notion.

The aim of this work is to study integrality of logsymplectic structure. Of course, we first use the notion of Lie-Rinehart to introduce the algebraic versus of logsymplectic structure. Secondly, we use the notion of logarithmic differential operator to define Dirac principle of prequantization of such structures. Since each $2 n$ dimensional logsymplectic manifold has $2 n-2$ dimensional leaves, we study the impact of the integrality of such leaves on the all manifold.

The main result of this paper is as follow:

Theorem 2.4 If the divisor $D$ is free and irreducible hypersurface of $X$ and the sheaf of logarithmic 1-form is generated by closed forms, then for all logarithmic 2-form $\omega$, the following conditions are equivalent 
a) $\omega=\frac{d h}{h} \wedge \psi+\eta$ is integral.

b) $\psi$ is exact and there is integral element $\left[\omega_{0}\right]$ of $\in H^{2}(X, \mathbb{C})$ such that $\left[\omega_{0}\right]=[\eta]$

The structure of the paper is as follows:

1. [Section 1] It is devoted to algebraic formalism of the notion of logsymplectic structure. Using the notion of Lie-Rinehart algebra, we define the notion of logsymplectic structures and we prove in Proposition 2.11 that such structures induce two Lie-structure on the base algebra. we also prove that Poisson structure associated to logsymplectic structure are logarithmic Poisson structure. We give two examples of logsymplectic structures and one example of non logsymplectic structure.

2. [Section 2] In this section, we recall the notion of logsymplectic manifold and we prove the main theorem of the note.

3. [Section 3] This section is devoted to the interpretation of Dirac principle for logsymplectic manifold. Of cause, we introduce the notion of logarithmic differential operator and we prove in Proposition 4.14 that the module of logarithmic differential operator is a Lie-Rinehart algebra. We also prove that the latter is central extension of logarithmic vector fields along the sheaf of holomorphic functions of complex manifold with free divisor.

4. [Section 4] We use extension of sheaf to study integrality condition of logsymplectic algebra.

5. [Section 5] In this section, we introduce Lie-algebroide formalism to study integrality of logsymplectic structures.

\section{Lie-Rinehart-logsymplectic Algebras and Associated Lie Brackets}

Throughout this section, $k$ denotes a field of characteristic 0 and $\mathcal{A}$ a commutative $k$-algebra with unity 1 .

Let $\operatorname{Der}_{\mathcal{A}}$ be the $\mathcal{A}$-module of $k$-derivations of $\mathcal{A}$ and $\Omega_{\mathcal{A}}$ the $\mathcal{A}$-module of formal differentials of $\mathcal{A}$. It is proved in (Huebschmann, J. (2013)) that $\Omega_{\mathcal{A}}$ is generated by $\{d a, a \in \mathcal{A}\}$ together with the relations

$$
d(a b)=a d b+b d a, \quad d(a+b)=d a+d b, \quad d(\lambda a)=\lambda d a \quad a, b \in \mathcal{A}, \lambda \in k ;
$$

where $d$ is the canonical derivation associated to $\Omega_{\mathcal{F}}$. An element $D$ of $D e r_{\mathcal{A}}$ is said to be logarithmic along an ideal $I$ of $\mathcal{A}$ if $D(I) \subset I$. We denote by $\operatorname{Der}_{\mathcal{A}}(\log I)$ the set of derivations of $\mathcal{A}$, logarithmic along $I$. By definition, $\operatorname{Der}_{\mathcal{A}}(\log I)$ is a sub-Lie algebra of $\operatorname{Der}_{\mathcal{A}}$. Let $S=\left\{u_{1}, \ldots, u_{p}\right\}$ be a subset of $p$ nonzero and nonunit elements of $\mathcal{A}$. An element $D$ of $\operatorname{Der}_{\mathcal{A}}$ is said to be logarithmic principal along the ideal generated by $S$ if for all $u_{i} \in S, D\left(u_{i}\right)$ is an element of the ideal $u_{i} \mathcal{A}$ generated by $u_{i}$. We denote by $\widehat{D e r_{\mathcal{A}}(\log I)}$ the set of derivations of $\mathcal{A}$ which are logarithmic principals along the ideal $I$ of $\mathcal{A}$ generated by $S$. $\overline{\operatorname{Der} \mathcal{A}(\log I)}$ is a sub-Lie algebra of $\operatorname{Der}_{\mathcal{A}}(\log I)$. We denote $\Omega_{\mathcal{A}}(\log I)$ the $\mathcal{A}$-module generated by $\left\{\frac{d u_{i}}{u_{i}} ; u_{i} \in S\right\} \cup \Omega_{\mathcal{A}}$.

We have the following:

Lemma 2.1. Let $I$ be the ideal $\mathcal{A}$ generated by $S=\left\{u_{1}, \ldots ; u_{p}, u_{i} \in \mathcal{A} ; 1 \leq i \leq p\right\}$. The $\mathcal{A}$-module $\widehat{\text { Der }} \widehat{r_{\mathcal{A}}(\log I) \text { of }}$ derivations of $\mathcal{A}$ logarithmic principal along $I$ is the dual over $\mathcal{A}$ of $\Omega_{\mathcal{A}}(\log I)$.

We recall that a Lie-Rinehart algebra over $\mathcal{A}$ is a pair $(L, \rho)$; where $L$ is Lie algebra which is also an $\mathcal{A}$-module and $\rho: L \rightarrow \operatorname{Der}_{k}(\mathcal{A})$ is Lie algebras homomorphism which satisfies the following equality.

$$
\left[l_{1}, a l_{2}\right]=\rho\left(l_{1}\right)(a) \cdot l_{2}+a\left[l_{1}, l_{2}\right]
$$

for all $a \in \mathcal{A}, l_{1} ; l_{2} \in L$.

Remark 2.2. The notion of Lie-Rinehart algebra is particular case of a more general notion called P-Lie-Rinehart algebra; where $P$ is an $\mathcal{A}$-algebra. More explicitly, a $P$-Lie-Rinehart algebra is a pair $(L, \rho)$ formed by a $P$-module $L$ and $\mathcal{A}$-linear map $\rho: L \longrightarrow$ Diff $_{1}(P)$ which is also a Lie-algebras homomorphism satisfies (1) for all $a \in P$ and $l_{1}, l_{2} \in L$. Where Diff $(P)$ denote the $\mathcal{A}$-module of first differentials operators of $P$. In the next section, we will recall the definition and give its logarithmic counterpart. 
It follows from this definition that $\operatorname{Der}_{\mathcal{H}}$ endowed with identity is Lie-Rinehart algebra. On the other hand, we can easily prove that the inclusion map of $\operatorname{Der}_{\mathcal{A}}(\log I)$ in $\operatorname{Der}_{\mathcal{A}}$ is a structure of Lie-Rinehart algebra on $\operatorname{Der}_{\mathcal{A}}(\log I)$.

Let $(L, \rho)$ be a Lie-Rinehart algebra over $\mathcal{A}$. By definition, the associated structure $\rho$ is also a representation of the $\mathcal{A}$ module $L$; by derivations of $\mathcal{A}$. For all $q \in \mathbb{N}$, a $q$-linear alternating mapping of $L$ into $\mathcal{A}$ is called a $q$-dimensional cochain. The set of $q$-cochains form a space $\mathcal{A} \operatorname{alt}^{q}(L, \mathcal{A})$. We define a linear mapping $d_{\rho}: \mathcal{L}$ alt ${ }^{q}(L, \mathcal{A}) \longrightarrow \mathcal{L}$ alt ${ }^{q+1}(L, \mathcal{A})$ by the formula

$$
\begin{aligned}
& d_{\rho} f\left(x_{0}, \ldots, x_{q}\right)=\sum_{i=1}^{p}(-1)^{i+1} \rho\left(x_{i}\right)\left(f\left(x_{0}, \ldots, \hat{x}_{i}, \ldots, x_{q}\right)\right) \\
& \quad+\sum_{i<j}(-1)^{i+1} f\left(\left[x_{i}, x_{j}\right], x_{0}, \ldots, \hat{x}_{i} \ldots, \hat{x}_{j}, \ldots, x_{q}\right)
\end{aligned}
$$

Lemma 2.3. For all Lie-Rinehart algebra $(L, \rho)$, we have $d_{\rho} \circ d_{\rho}=0$

We adopt the following definitions.

Definition 2.4. The cohomology of the complex $\left(\mathcal{L} a l t^{q}(L, \mathcal{A}), d_{\rho}\right)$ is called Lie-Rinehart cohomology of $L$.

The following is easy to prove.

Proposition 2.5. If $\operatorname{Der}_{\mathcal{A}}$ and $\operatorname{Der}_{\mathcal{A}}(\log I)$ are respectively the Lie-Rinehart algebra of derivations of $\mathcal{A}$ and of logarithmic derivations of $\mathcal{A}$, then

(i) The Lie-Rinehart cohomology of $\operatorname{Der}_{\mathcal{A}}$ is the De Rham cohomology.

(ii) The Lie-Rinehart cohomology of $\operatorname{Der}_{\mathcal{A}}(\log I)$ is the logarithmic De Rham cohomology

Follows J. Huebschmann in (Huebschmann, J. (2013)), we can now introduce the following notion.

Definition 2.6. A structure of Lie-Rinehart-Poisson on a Lie-Rinehart algebra $(L, \rho)$ is an alternating 2-form $\mu$

$$
\mu: L \times L \rightarrow \mathcal{A}
$$

such that

$$
d_{\rho} \mu=0 .
$$

If $\mu$ is a structure of Lie-Rinehart-Poisson on $(L, \rho)$,then $(L, \rho, \mu)$ is called a Lie-Rinehart-Poisson algebra. The notion of Lie-Rinehart-Poisson algebra is a part of more general notion defined by I. Krasil'shchik in (Krasil'shchik, I. (1988)) He called it canonical algebra. We denote by $L^{*}$ the algebraic dual over $\mathcal{A}$ of a Lie-Rinehart algebra $L$.

Definition 2.7. see (Huebschmann, J. (2013)) An Lie-Rinehart-Poisson algebra $(L, \rho, \mu)$ is said to be a Lie-RinehartPoisson-symplectic algebra if $\mu$ is nondegenerated i.e;

$$
L \rightarrow L^{*}, x \mapsto i_{x} \mu
$$

is an isomorphism of $\mathcal{A}$-modules. Where for all $x \in L$,

$$
i_{x}: \mathcal{L} \operatorname{alt}(L, \mathcal{A}) \longrightarrow \mathcal{L} \operatorname{alt}(L, \mathcal{A})
$$

such that for all $x_{1}, \ldots, x_{q-1} \in L$; and for all $f \in \mathcal{L}$ alt ${ }^{q}(L, \mathcal{A})$ we have

$$
\left(i_{x} f\right)\left(x_{1}, \ldots, x_{q-1}\right)=f\left(x, x_{1}, \ldots, x_{q-1}\right) .
$$

Remark 2.8. If $X$ is a smooth manifold then

(i) Every Poisson structure on $X$ is a Lie-Rinehart-Poisson structure on $\Omega_{X}$ with Lie-Rinehart structure the Hamiltonian map.

(ii) Every symplectic structure on $X$ is a Lie-Rinehart-Poisson-symplectic on the $O_{X}$-module of smooth vector fields on $X$

It follows from the definition that $\operatorname{Der}_{\mathcal{A}}(\log I)$ is a Lie-Rinehart algebra; with inclusion as structure. 
Definition 2.9. Lie-Rinehart-Poisson-symplectic structure on $\operatorname{Der}_{\mathcal{A}}(\log I)$ are called logsymplectic structure.

In particular, when $\mathcal{A}$ is the algebra of holomorphic functions on a $2 n$ dimensional complex manifold $X$, a logsymplectic structure on $\mathcal{A}$ is algebraic analogous logsymplectic form on $X$ (see (Goto, R. (2002))). According to above the definition, logsymplectic structure are 2-forms on $\operatorname{Der}_{\mathcal{H}}(\log I)$ which are closed under the De Rham differential. Since $\operatorname{Der}_{\mathcal{A}}(\log I)$ is sub-module of $\operatorname{Der}_{\mathcal{A}}$ its algebraic dual over $\mathcal{A}$ is bigger than $\operatorname{Der}_{\mathcal{A}}$ one's; which is not well defined since, in general the bi-dual of $\Omega_{\mathcal{A}}$ is not $\Omega_{\mathcal{A}}$. In (Saito, K. (1980)), the author proves that the sheaf of logarithmic forms and the sheaf of logarithmic vector field on final dimensional complex manifold are dual each other.

Let $\mu$ be a logsymplectic structure. Since $\mu$ is non degenerated, its contraction by logarithmic derivation induce an isomorphism of $\mathcal{A}$-modules between $\operatorname{Der}_{k}(\log I)$ and its dual $\operatorname{Der}_{k}(\log I)^{*}$ which is the module of logarithmic forms (see (Saito, K. (1980))). Therefore, for all $a \in \mathcal{A}$, there is an unique $\delta_{a} \in \operatorname{Der}_{k}(\log I)$ such that:

$$
i_{\left(\delta_{a}\right)} \mu=d a .
$$

$a$ is called logarithmic Hamiltonian element and $\delta_{a}$ is called logarithmic Hamiltonian field. For all $a, b \in \mathcal{A}$, consider:

$$
\{a, b\}=-\mu\left(\delta_{a}, \delta_{b}\right)
$$

We have the following proposition.

Proposition 2.10. (Dongho, J.(2012) Let $\mu$ be a logsymplectic structure on $\mathcal{A}$. The following bracket

$$
\{a, b\}=-\mu\left(\delta_{a}, \delta_{b}\right)
$$

is a well defined logarithmic Poisson structure on $\mathcal{A}$

In the sequel, we suppose that $I$ is generated by $S=\left\{u_{1}, \ldots, u_{q}\right\}$ and $\mu$, is a logsymplectic structure. Then for all $u_{i} \in S$ there exist a unique $\tilde{\delta}_{u_{i}} \in \operatorname{Der}_{k}(\log I)$ such that

$$
i_{\tilde{\delta}_{u_{i}}} \mu=\frac{d u_{i}}{u_{i}} .
$$

But since $d u_{i} \in \Omega_{\mathcal{A}} \subset \Omega_{\mathcal{A}}(\log I)$, there exist $\delta_{u_{i}}$ such that $i_{\delta_{u_{i}}} \mu=d u_{i}$. It is easy to prove that $\delta_{u_{i}}=u_{i} \tilde{\delta}_{u_{i}}$. We can then consider the following bracket:

$$
\{a, b\}_{\text {sing }}:=\left\{\begin{array}{ccc}
\frac{1}{u v}\{u, v\} & \text { if } & a=u, b=v \in S \\
\frac{1}{u}\{u, b\} & \text { if } & a=u \in S, b \in \mathcal{A}-S \\
\{a, b\} & \text { if } & a, b \in \mathcal{A}-S
\end{array}\right.
$$

By direct computation, we have the following proposition.

Proposition 2.11. A Logsymplectic structure $\mu$ on $\mathcal{A}$ induces two Lie structures $\{-,-\}$ and $\{-,-\}_{\text {sing }}$ such that for all $u, v \in I-0$,

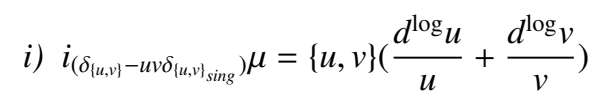

ii) $\{u v, a\}_{\text {sing }}=\{u+v, a\}_{\text {sing }} ; \forall a \in \mathcal{A}-I$.

iii) $\{a, b\}=\delta_{a}(b)$

iv) $\left[\delta_{a}, \delta_{b}\right]=\delta_{\{a, b\}}$

v) $\delta_{\{u, v\}}=u v\left[\tilde{\delta_{u}}, \tilde{\delta_{v}}\right]+\{u, v\}\left(\tilde{\delta_{v}}+\tilde{\delta_{u}}\right)$

Example 2.12. (Dongho, J.(2012) 
Let $\mathcal{A}=\mathbb{C}[x, y]$ the algebra of two variables polynomials and $I=x \mathcal{A}, J=y \mathcal{A}, K=x^{2} \mathcal{A}$ the ideal generated respectively by $x, y$ and $x^{2}$. We have:

$$
\Omega_{\mathcal{A}}(\log I) \cong<\frac{d x}{x}>_{\mathbb{C}[y]} \oplus \Omega_{\mathcal{A}} \cong \Omega_{\mathcal{A}}(\log K)
$$

and

$$
\Omega_{\mathcal{A}}(\log J) \cong<\frac{d y}{y}>_{\mathbb{C}[x]} \oplus \Omega_{\mathcal{H}} .
$$

Where $\left\langle U>_{B}\right.$ denotes the $B$-module generated by $U$ for all $U \subset L$ and $B \subset \mathcal{A}$. The following 2-forms

$$
\omega_{0}=\frac{d x}{x} \wedge d y, \omega_{1}=\frac{d y}{y} \wedge d x, \omega_{=} d x \wedge d y
$$

Are Lie-Rinehart-Poisson structure on $\operatorname{Der}_{\mathcal{A}}(\log I), \operatorname{Der}_{\mathcal{A}}(\log J)$ and $\operatorname{Der}_{\mathcal{A}}(\log K)$ respectively; since $d \omega_{0}=d \omega_{1}=d \omega_{2}=$ 0 . We recall that in this case, the Lie-Rinehart structure is just their inclusion in $D e r_{\mathcal{H}}$. The matrix of $\omega_{1}$ relative to the bases $\left(x \partial_{x}, \partial_{y}\right)$ of $\operatorname{Der}_{\mathcal{A}}(\log I)$ and $\left(\frac{d x}{x}, d y\right)$ of $\Omega_{\mathcal{A}}(\log I)$ is

$$
M_{\omega}=\left(\begin{array}{cc}
0 & -1 \\
1 & 0
\end{array}\right)
$$

It is also the matrix of $\omega_{2}$ relative to the bases $\left(\partial_{x}, y \partial_{y}\right)$ of $\operatorname{Der}_{\mathcal{H}}(\log J)$ and $\left(d x, \frac{d y}{y}\right)$ of $\Omega_{\mathcal{A}}(\log J)$. Since de determinant of $M_{\omega}$ is inversible element of the ring $\mathcal{A}$, we conclude that $\omega_{1}$ and $\omega_{2}$ are logsymplectic structures. On the other hand, the matrix of $\omega_{3}$ relative to the bases $\left(x \partial_{x}, \partial_{y}\right)$ of $\operatorname{Der}_{\mathcal{A}}(\log I)$ and $\left(\frac{d x}{x}, d y\right)$ of $\Omega_{\mathcal{A}}(\log I)$ is

$$
M_{\omega_{3}}=\left(\begin{array}{cc}
0 & -x \\
x & 0
\end{array}\right)
$$

and its determinant is $x^{2}$ which is not an inversible element of $\mathcal{A}$. Therefore, $\omega_{3}$ is a Lie-Rinehart-Poisson structure which is not a logsymplectic structure.

\section{Logsymplectic Manifold}

In this section we propose a geometric application of the concepts introduced in the above section. As we can see, the geometric analogous of Lie-Rinehart-logsymplectic structure correspond to logsymplectic structure. Throughout this section, $X$ a a final dimensional compact complex manifold with reduced divisor $D$. To define what is logsymplectic manifold, we need the notion of logarithmic forms which are extensively studied in algebraic geometry; see (Saito, K. (1980)). In addition to the assumptions made in (Saito, K. (1980)), we assume that $D$ is square free.

Let $\omega$ be a meromorphic q-form on $X$ with poles only in $D$. We suppose that $D:=\{z \in X, h(z)=0\}$ where $h$ is some holomorphic map.

$\omega$ is said to be logarithmic along $D$ if $h \omega$ and $d h \wedge \omega$ are holomorphic forms. As in (Saito, K. (1980)), we denote $\Omega_{X}^{q}(\log D)$ the sheaf of logarithmic q-forms on $X$.

Remark 3.1. (Dongho, J.(2012)) Let $X=\mathbb{C}^{2}$ and $D=\{0\} \times \mathbb{C}$. In canonical coordinate system $(x, y)$ of $X$, the divisor $D$ is the set of zero of the ideal I generated by $x$. But the only useful element of I which represent completely $D$ is $h=x$. Other element of ideal I are square free. It follows from our definition of logarithmic forms that $\frac{d x}{x^{2}}$ is not logarithmic 1-form since $x \frac{d x}{x^{2}}=\frac{d x}{x}$ which is not holomorphic 1-form. But according to K. Saito definition (See Definition 1.2 of (Saito, K. (1980)), $\frac{d x}{x^{2}}$ and dy are logarithmic forms. In addition, $\frac{d x}{x^{2}} \wedge d y=1 . \frac{d x \wedge d y}{x^{2}}$. Therefore, according to Theorem 1.8 of (Saito, K. (1980)), the system $\left\{\frac{d x}{x^{2}}, d y\right\}$ shall be a bases of $\Omega_{X}(\log D)$. But on the other hand, $\left\{\frac{d x}{x}, d y\right\}$ is free bases of $\Omega_{X}(\log D)$. Then there exist two holomorphic functions $a$ and $b$ such that $\frac{d x}{x^{2}}=a \frac{d x}{x}+b d y$; but this implies that ax $=1$ and $b=0$. Then $a=\frac{1}{x}$. Which is contradictory to our assumptions. So $\frac{d x}{x^{2}}$ is not logarithmic 1-form.

It follows from this remark that the assumption that $D$ shall be square free is necessary. 
Definition 3.2. (Goto, R. (2002)) A pair $(X, D)$ is logsymplectic manifold if there is a logarithmic 2-form $\Omega_{X}^{2}(\log D)$ satisfying

$$
d \omega=0, \overbrace{\omega \wedge \omega \wedge \ldots \wedge \omega}^{\text {ntimes }} \neq O \in H^{0}\left(X, \Omega^{2 n}[D]\right)
$$

One important notion related to logarithmic forms is associated residue form. According to Theorem 1.1 of (Saito, K. (1980)), if its is a $n$-dimensional complex manifold and $\omega$ is a logarithmic q-form, then there exist an holomorphic function $g$ such that

a) $\operatorname{dim}_{\mathbb{C}} D \cap\{z \in X ; g(z)=0\} \leq n-2$

b) $g \omega=\frac{d h}{h} \wedge \psi+\eta$. Where $\psi$ and $\eta$ are holomorphic form.

Thus, the logarithmic forms may have poles outside of $D$. One form $\operatorname{Re}(\omega)=\frac{\psi}{g}$ is called residue form of $\omega . D$ is said to be free if the germs of $\Omega_{X}(\log D)$ at each point of $D$ is free. When $D$ is free, $\Omega_{X}^{q}(\log D)=\wedge^{q} \Omega_{X}(\log D)$ in such a way that residues of all q-form is holomorphic on $X$ if $h$ is irreducible and the residues of all element of $\Omega_{X}(\log D)$ is holomorphic. In such cases, global sections of $\Omega_{X}^{2}(\log D)$ are in the form $\frac{d h}{h} \wedge \psi+\eta$ where $\psi$ and $\eta$ are holomorphic forms.

Let us denote $H^{*}(X, \mathbb{C})$ the De Rham cohomology of $X$. According to De Rham Theorem, it is isomorphic to the cohomology of the complex of holomorphic forms of $X$. Since the latter is sub-complex of the complex of logarithmic forms, there is an homomorphism $p: H^{*}(X, \mathbb{C}) \rightarrow H^{*}\left(X, \Omega_{X}^{*}(\log D)\right)$ where $H^{*}\left(X, \Omega_{X}^{*}(\log D)\right)$ is the cohomology group of the logarithmic De Rham complex. So we have the following sequences

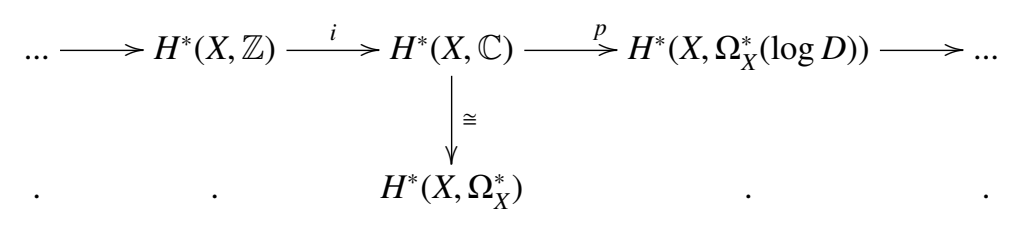

Definition 3.3. An element $[\omega]$ of $H^{2}\left(X, \Omega_{X}^{*}(\log D)\right)$ is said to be integral if $[\omega]=p \circ i\left(\left[\omega_{0}\right]\right)$, for some $\left[\omega_{0}\right] \in H^{*}(X, \mathbb{Z})$.

With those tools, we can prove the following result.

Theorem 3.4. If the divisor $D$ is free and irreducible hypersurface of $X$ and the sheaf of logarithmic 1-form is generated by closed forms, then for all logarithmic 2-form $\omega$, the following conditions are equivalent

a) $\omega=\frac{d h}{h} \wedge \psi+\eta$ is integral.

b) $\psi$ is exact and there is integral element $\left[\omega_{0}\right]$ of $\in H^{2}(X, \mathbb{C})$ such that $\left[\omega_{0}\right]=[\eta]$

Proof. Suppose that $\omega$ is integral. Then there exist $\left[\omega_{1}\right] \in H^{2}(X, \mathbb{Z})$ such that $[\omega]=p \circ i\left[\omega_{1}\right]$.

Let us prove that $\left[\omega_{0}\right]=i\left(\left[\omega_{1}\right]\right)$. By definition, $\omega_{0}$ is integral. Since $\omega$ is integral, $[\omega]=p \circ i\left[\omega_{1}\right]$. Then there is a logarithmic 1-form $\alpha=\alpha_{0} \frac{d h}{h}+\alpha_{1}$ such that $\omega-\omega_{0}=d \alpha$. Then $-d \alpha_{0}=\psi$ and $\eta=\omega_{0}+d \alpha_{1}$. But $d \omega=0$ iff $\frac{d h}{h} \wedge d \psi=-d \eta$. therefore, $d \psi=0$ and $d \eta=0$.

conversely, if $\omega_{0}+d \lambda=\eta$ and $\psi=d \beta$ with $\omega_{0}$ integral, then

$$
\begin{aligned}
\omega & =d\left(-\beta \frac{d h}{h}\right)+\eta \\
& =\omega_{0}+d \lambda+d\left(-\beta \frac{d h}{h}\right) \\
& =\omega_{0}+d\left(\lambda-\beta \frac{d h}{h}\right)
\end{aligned}
$$

Therefore, $[\omega]=\left[\omega_{0}\right]$. 
Let us denote by $D_{\text {sing }}$ the singular part of $D$ and $D_{\text {red }}$ the smooth part. The proof of the following is essentially the same as the proof of Darboux Theorem in symplectic geometry.

Lemma 3.5. (Goto, R. (2002)) (Log Darboux Theorem). Let (X,D) be a log symplectic manifold with a logarithmic symplectic form $\omega$, where $D$ is reduced divisor. There exist holomorphic coordinates $\left(z_{0}, z_{1}, \ldots, z_{2 n-1}\right)$ of a neighborhood of each smooth point of $D_{\text {red }}$ such that $\omega$ is given by

$$
\omega=\frac{d z_{0}}{z_{0}} \wedge+\ldots+d z_{2 n-2} \wedge d z_{2 n-1}
$$

where $\left\{z_{0}=0\right\}=D$. we refer to these coordinates as log Darboux coordinates.

It follow from this result that the residue 1-form of a logarithmic 2-form $\omega$ if $d z_{1}$ in the log Darboux coordinates. We have then an integrable distribution by setting $\left\{\delta \in T_{D_{\text {red }}}, \operatorname{Re}(\omega)(\delta)=0\right\}$. Therefore, we have $2 n$-2-dimensional leaves on $D_{\text {red }}$. More explicitly, we have the following result.

Lemma 3.6. (Goto, R. (2002)). The log symplectic form $\omega$ defines a symplectic structure on each leaf.

It follows from this lemma that in a complement of 2-dimensional sub-manifold of $X$, logsymplectic forms are symplectic.

\section{Logarithmic Lie-Rinehart Differential Operators}

In this paragraph, $(X, \omega, D)$ is logsymplectic manifold and $\mathcal{E}$ a locally free $O_{X}$-module of rank 1 and $D=\{h=0\}$ a divisor of $X$.

\subsection{Logarithmic Connection}

The notion of logarithmic connection is original in the work of P. Deligne when he formulated and proved the theorem establishing a Riemann-Hilbert correspondence between monodromy groups and Fuchsian systems of integrable partial equations or flat connections on complex manifolds. He also gives a treatment of the theorem of Griffiths which states that the Gauss-Manin or Picard-Fuchs systems of of differential equations are regular and singular.

Definition 4.1. Let $\mathcal{M}$ be an $O_{X}$-module. A connection on $\mathcal{M}$ with logarithmic poles along $D$ is a $\mathbb{C}$-linear homomorphism $\nabla$

$$
\nabla: \mathcal{M} \rightarrow \Omega_{X}^{1}(\log D) \otimes \mathcal{M}
$$

that satisfies Leibniz's identity:

$$
\nabla(f m)=d f \cdot m+f \nabla(m)
$$

where $d$ is the exterior derivative over $O_{X}$.

This is equivalent to a linear map $\Delta: \operatorname{Der}_{X}(\log D) \rightarrow \operatorname{End}(\mathcal{E})$ satisfy the following

$$
\triangle_{X}(f s)=f \triangle_{X} s+X(f) s
$$

If $\nabla$ is logarithmic connection $K_{\nabla}$ will denote its curvature and the pair $(\mathcal{M}, \nabla)$ will refer to logarithmic connection on a locally free $O_{X}$-module of rank $1 \mathcal{M}$.

Lemma 4.2. If $(\mathcal{M}, \nabla)$ is rank one connection on $X$, then for all closed 1-form $\tau \in H^{0}\left(X, \Omega_{X}^{1}(\log D)\right),(\mathcal{M}, \nabla+\tau \otimes$ id $)$ is a connection with curvature form $K=K_{\nabla}$

Proof. Suppose that $\nabla$ is define by

$$
\nabla(s)=\sigma \otimes s
$$

for a giving nowhere vanish section $s$

$$
\begin{aligned}
(\nabla+\tau \otimes i d)(s) & =\nabla(s)+\tau \otimes s \\
& =\sigma \otimes s+\tau \otimes s \\
& =(\sigma+\tau) \otimes s
\end{aligned}
$$

And

$$
\begin{aligned}
(\nabla+\tau \otimes i d)(f s) & =\nabla(f s)+\tau \otimes i d(f s) \\
& =d f \otimes s+f \sigma \otimes s+f \tau \otimes s \\
& =d f \otimes s+f(\nabla+\tau \otimes i d) s
\end{aligned}
$$


Let $s_{0} \in H^{0}\left(U_{i}, \mathcal{M}\right)$ such that $0 \notin s_{0}\left(U_{i}\right)$. There exist $\sigma \in H^{0}\left(U_{i}, \Omega_{X}^{1}(\log D)\right)$ such that $\nabla s_{0}=\sigma \otimes s_{0}$. Then $K_{\nabla}=d \sigma$. Let $p$ be a point of $D$ and $\left(z_{\lambda}^{i}\right)$ a logarithmic coordinate system along $D$ at $p$.

$$
\sigma=\sum_{i=1}^{r} a_{i} \frac{d z_{\lambda}^{i}}{z^{i} \lambda}+\sum_{i=r+1}^{2 n} a_{i} d z_{\lambda}^{i}
$$

where $a_{i} \in H^{0}\left(X, O_{X}\right)$. Therefore, we deduce that:

Lemma 4.3. Let $D$ be a normal crossing divisor and $\alpha \in H^{0}\left(X, \Omega_{X}(\log D)\right)$. If $d \alpha=0$ then the residue of $\alpha$ is constant on any component of singular locus of $D$. Any such form with at least one nonzero residue admits representation

$$
\alpha=\sum_{j=1}^{r} \alpha_{i} \frac{d f_{j}}{f_{j}}, \quad \alpha_{1}, \ldots, \alpha_{r} \in \mathbb{C}
$$

Proof. Let $p$ be a point of $D$ and $U_{\lambda}$ an open coordinate neighborhood of $p$. We have: $\alpha=\operatorname{Res}(\alpha) \frac{d h_{p}}{h_{p}}+\alpha_{\text {reg }}$ where $\operatorname{Res}(\alpha)$ is the residue of $\alpha$. But $d \alpha=0$ imply $d(\operatorname{Res} \alpha)=O$; since Res commute with $d$. However, from Theorem 2.9 in (K. Saito, 1980), $\operatorname{Res}\left(\Omega_{X}^{1}(\log D)\right)=O_{X}$, therefore $d \operatorname{Res}(\alpha)=0$ imply $\operatorname{Res} \alpha \in \mathbb{C}$.

Proposition 4.4. $K_{\nabla}=0$ if and only if $\sigma=\sum_{i=1}^{r} a_{i} \frac{d z_{\lambda}^{i}}{z_{\lambda}^{i}}$ with $a_{i} \in \mathbb{C}$.

Proof. $K_{\nabla}=d \sigma$; where $\sigma$ is the connection one form of $\nabla$. The result is then a consequence of Lemma 4.3

Definition 4.5. Let $(\mathcal{M}, \nabla)$ and $(\mathcal{N}, \delta)$ be two connections. An homomorphism from $(\mathcal{M}, \nabla)$ to $(\mathcal{N}, \delta)$ is a sheaf homomorphism $\varphi: \mathcal{M} \rightarrow \mathcal{N}$ such that the following diagram commute.

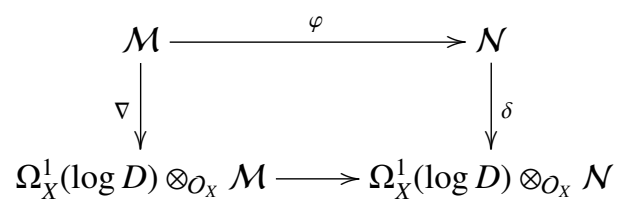

Definition 4.6. Let $(\mathcal{M}, \nabla)$ be a connection on $X^{*}=X-D$. A meromorphic prolongation of $(\mathcal{M}, \nabla)$ is a meromorphic connection $(\overline{\mathcal{M}}, \bar{\nabla})$ on $X$ such that the restriction is an isomorphism.

\subsection{Module of Logarithmic Differential Operator}

Let $\mathcal{A}$ be a commutative ring. For any pair of $\mathcal{A}$-modules $M, N$ we define module $\operatorname{Dif} f_{\mathcal{A}}^{k}(M, N)$ inductively by putting

1. $\operatorname{Diff}_{\mathcal{A}}^{0}(M, N)=\operatorname{Hom}_{\mathcal{H}}(M, N)$

2. $\operatorname{Diff}_{\mathcal{A}}^{k}(M, N)=$

$\left\{\right.$ additive maps $u: M \rightarrow N$ s.t $\forall a \in \mathcal{A} \quad a u-u a \in \operatorname{Diff}_{\mathcal{A}}^{k-1}(M, N)$ \}

Elements of $\operatorname{Diff}_{\mathcal{A}}^{k}(M, N)$ are called $k$-order differential operator from $M$ to $N$. We note $\operatorname{Dif} f_{\mathcal{A}}^{k}(M)$ for $\operatorname{Diff} f_{\mathcal{A}}^{k}(M, M)$. Replacing $M$ by $\mathcal{E}$, the above definition becomes;

Definition 4.7. An r-order differential operator on $\mathcal{E}$ is a $\mathbb{C}$-linear map $\varphi: \mathcal{E} \rightarrow \mathcal{E}$ such that $s \mapsto \varphi(f s)-f \varphi(s)$ is an $(r-1)$-order differential operator on $\mathcal{E})$; for all $f \in O_{X}$

In the previous paragraph, we see that each logarithmic connection induces a morphism $\triangle$ on $\operatorname{Der}_{X}(\log D)$ such that for all $f \in O_{X}$ and $X \in \operatorname{Der}_{X}(\log D)$, we have $\Delta_{X}(f s)-f \Delta_{X}(s)=X(f) s$. It follows that $\triangle_{X} \in \operatorname{End}(\mathcal{A})$ and the map

$\triangle_{X}^{f}: s \mapsto \triangle_{X}(f s)-f \triangle_{X}(s)$ is zero order differential operator on $\mathcal{E}$ and $\triangle_{X}^{f}(h) \in h O$. Therefore, there is an unique $f_{h} \in O$ such that $h^{-1}\left[\triangle_{X}^{f}(h)\right] s=f_{h} s$ for all $s \in \mathcal{E}$. In other words, $h^{-1}\left[\triangle_{X}^{f}(h)\right]$ is zero order operator. This motivate the following definition.

Definition 4.8. An r-order differential operator $\varphi$ is logarithmic along $D$ if $s \mapsto[\varphi(h s)-h \varphi(s)] h^{-1}$ is an (r-1)-order differential operator on $\mathcal{E}$. 
Notation 4.9. We denote Diff ${ }^{r}(\mathcal{E})$ the set of $r$-order differential operators and Diff $f_{\log }^{r}(\mathcal{E})$ is the subset of $r$-order differential operators logarithmic along $D$

According to what precedes, $\triangle_{X} \in \operatorname{Diff}_{\log }^{1}(\mathcal{E})$; for all $X \in \operatorname{Der}_{X}(\log D)$.

Lemma 4.10. Let $\varphi$ be a first order differential operator logarithmic along $D$, for all sections $f$ of $O_{X}$, There exists unique $\tilde{f} \in O_{X}$ such that $[\varphi(f s)-f \varphi(s)]=m_{\tilde{f}} s$.

Proof. $[s \mapsto \varphi(f s)-f \varphi(s)] \in \operatorname{Diff} f_{\log }^{0}(\mathcal{E})$ then there exist $\tilde{f} \in O_{X}$ such that $[\varphi(f s)-f \varphi(s)]=m_{\tilde{f}} s$. If $g$ is another section of $O_{X}$ such that $[\varphi(f s)-f \varphi(s)]=m_{g} s$, Then $\tilde{f} s=g s$ for all $s \in \mathcal{E}$; i.e; $\tilde{f}=g$

Corollary 4.11. If $\varphi$ is a first order operator logarithmic along $D$ then $\tilde{h} \in h O_{X}$

Proof. For all $s \in \mathcal{E}, \varphi(h s)-h \varphi(s)=\tilde{h} s$ and there exist $g \in O_{X}$ such that $\varphi(h s)-h \varphi(s)=h g s$. Therefore, $(\tilde{h}-h g) s=0$ for all $s$.

It follows that any first order differential operator logarithmic along $D, \varphi$ gives rise to a map $\sigma_{\varphi}: O_{X} \rightarrow O_{X}$ defined by $\sigma_{\varphi}(f)=\tilde{f}$ such that $[\varphi(f s)-f \varphi(s)]=\tilde{f} s$ for all $s \in \mathcal{E}$.

Lemma 4.12. For all $\varphi \in \operatorname{Diff}_{\log }^{1}(\mathcal{E}), \sigma_{\varphi} \in H^{0}\left(X, \operatorname{Der}_{X}^{1}(\log D)\right)$

Proof.

$$
\begin{aligned}
\sigma_{\varphi}(f . g) s & =\varphi(f(g s)-f g \varphi(s) \\
& =\sigma_{\varphi}(f)(g s)+f \varphi(g s)-f g \varphi(s) \\
& =\sigma_{\varphi}(f)(g s)+f(\varphi(g s)-g \varphi(s)) \\
& =\left(\sigma_{\varphi}(f) g+f \sigma_{\varphi}(g)\right) s
\end{aligned}
$$

On the other hand, we have;

$$
\begin{aligned}
\sigma_{\varphi}(h) s & =\varphi(h s)-h \varphi(s) \\
& =h m_{\tilde{h}}(s)
\end{aligned}
$$

Then $\left(\sigma_{\varphi}(h)-h m_{\tilde{h}}\right) s=0$ for all $s$

Therefore, $\sigma_{\varphi}(h) \in h O_{X}$; i.e., $\sigma_{\varphi} \in H^{0}\left(X, \operatorname{Der}_{X}^{1}(-\log D)\right)$.

Proposition 4.13. Dif $f_{\log }^{1}(\mathcal{E})$ is closed under commutator.

Proof. Let $\varphi_{1}, \varphi_{2}$ be two sections of $\operatorname{Diff} f_{\log }^{1}(\mathcal{E})$;

We have:

$$
\begin{aligned}
\varphi_{1} \varphi_{2}(f s) & =\varphi_{1}\left(f \varphi_{2}(s)+\bar{f}^{2} s\right) \\
& =f \varphi_{1}\left(f \varphi_{2}(s)+\varphi_{1}\left(\bar{f}^{2} s\right)\right) \\
& =f \varphi_{1}\left(\varphi_{2}(s)\right)+\bar{f}^{1} \varphi_{2}(s)+\bar{f}^{2} \varphi_{1}(s)+\overline{\bar{f}}^{2}{ }^{1} s
\end{aligned}
$$

On the same way, we obtain:

$\varphi_{2} \varphi_{1}(f s)=f \varphi_{2}\left(\varphi_{1}(s)\right)+\bar{f}^{2} \varphi_{1}(s)+\bar{f}^{1} \varphi_{2}(s)+\overline{\bar{f}}^{1}{ }^{2} s$ therefore $\varphi_{1} \varphi_{2}(f s)-\varphi_{2} \varphi_{1}(f s)-f\left(\varphi_{1} \varphi_{2}-\varphi_{2} \varphi_{1}\right)(s)=\left(\overline{\bar{f}}^{1}-\overline{\bar{f}}^{2}\right) s$ On the other hand, since $\varphi_{1}, \varphi_{2}$ are sections of $\operatorname{Diff}_{\log }^{1}(\mathcal{E})$, there exist $h_{1}, h_{2} \in O_{X}$ such that: $\left[\varphi_{2}(h s)-h \varphi_{2}(s)\right] \frac{1}{h}=h_{2} s$ and $\left[\varphi_{1}(h s)-h \varphi_{1}(s)\right] \frac{1}{h}=h_{1} s$ i.e; $\bar{h}^{2}=h h_{2}$ and $\bar{h}^{1}=h h_{1}$. On the same way, there exist $h_{21}, h_{12} \in O_{X}$ such that $\overline{\bar{h}}^{1^{2}}=h h_{12}$ and $\overline{\bar{h}}^{2}{ }^{1}=h h_{21}$. Therefore, $\varphi_{1} \varphi_{2}(h s)-\varphi_{2} \varphi_{1}(h s)-h\left(\varphi_{1} \varphi_{2}-\varphi_{2} \varphi_{1}\right)(s)=\left(\overline{\bar{h}}^{2}-\overline{\bar{h}}^{2}\right) s=h\left[h_{21}-h_{12}\right] s$

Proposition 4.14. Dif $f_{\log }^{1}(\mathcal{E})$ is a logarithmic Lie-Rinehart algebra.

Proof. According to above results, we have the following map.

$$
\begin{array}{ccc}
\operatorname{Diff}_{\log }^{1}(\mathcal{E}) & \rightarrow & \operatorname{Der}_{X}(\log D) \\
\varphi & \mapsto & \sigma_{\varphi}
\end{array}
$$

For all $f \in O_{X}, s \in \mathcal{E}$, we have: 


$$
\begin{array}{rlr}
\sigma_{\left[\varphi_{1}, \varphi_{2}\right]}(f) s= & {\left[\varphi_{1}, \varphi_{2}\right](f s)-f\left[\varphi_{1}, \varphi_{2}\right](s)} \\
= & \varphi_{1} \varphi_{2}(f s)-\varphi_{2} \varphi_{1}(f s)-f \varphi_{1} \varphi_{2}(s)+\varphi_{2} \varphi_{2}(s) \\
= & \varphi_{1}\left(\sigma_{\varphi_{2}}(f) s+f \varphi_{2}(s)\right)-\varphi_{2}\left(\sigma_{\varphi_{1}}(f) s+f \varphi_{1}(s)\right)-f\left[\varphi_{1}, \varphi_{2}\right] s & \\
= & \varphi_{1}\left(\sigma_{\varphi_{2}}(f) s\right)+\varphi_{1}\left(f \varphi_{2}(s)\right)-\varphi_{2}\left(\sigma_{\varphi_{1}}(f) s\right)-\varphi_{2}\left(f \varphi_{1}(s)\right)-f\left[\varphi_{1}, \varphi_{2}\right] s \quad \text { On the other hand, for all } \varphi_{1}, \varphi_{2} \in \\
= & \sigma_{\varphi_{1}}\left(\sigma_{\varphi_{2}}(f)\right) s+\sigma_{\varphi_{2}}(f) \varphi_{1}(s)+\sigma_{\varphi_{1}}(f) \varphi_{2}(s)+f \varphi_{1}\left(\varphi_{2}(s)\right)- & \\
& \sigma_{\varphi_{2}}\left(\sigma_{\varphi_{1}}(f)\right) s-\sigma_{\varphi_{1}}(f) \varphi_{2}(s)-\sigma_{\varphi_{2}}(f) \varphi_{1}(s)-f \varphi_{2}\left(\varphi_{1}(s)\right)-f\left[\varphi_{1}, \varphi_{2}\right] s & \\
= & {\left[\sigma_{\varphi_{1}}, \sigma_{\varphi_{2}}\right](f) s}
\end{array}
$$

$D i f f_{\log }^{1}(\mathcal{E}), f \in O$ and $s \in \mathcal{E}$ we have:

$$
\begin{aligned}
{\left[\varphi_{1}, f \varphi_{2}\right] } & =\varphi_{1}\left(f \varphi_{2}(s)\right)-\left(f \varphi_{2}\right)\left(\varphi_{1}(s)\right) \\
& =f \varphi_{1}\left(\varphi_{2}(s)\right)+\sigma_{\varphi_{1}}(f)\left(\varphi_{2}(s)\right)-f \varphi_{2}\left(\varphi_{1}(s)\right) \\
& =\sigma_{\varphi_{1}}(f)\left(\varphi_{2}(s)\right)+f\left[\varphi_{1}, \varphi_{2}\right]
\end{aligned}
$$

From above results, we deduce the following exact sequence of Lie-Rinehart algebras

$$
0 \longrightarrow O_{X} \stackrel{m}{\longrightarrow} \operatorname{Diff}_{\log }^{1}(\mathcal{E}) \stackrel{\sigma}{\longrightarrow} \operatorname{Der}_{X}(\log D) \longrightarrow 0
$$

\section{Prequantization}

Let $\mathcal{H}^{\log }(\mathcal{A}, I):=\left\{\delta \in \operatorname{Der}_{k}(\log I) ; \exists a \in \mathcal{A} ; \delta=\delta_{a}\right\}$. Clearly $\mathcal{H}^{\log }(\mathcal{A}, I)$ is sub-Lie-algebra of $\operatorname{Der}_{k}(\log I)$ and we have the following exact sequence of Lie-algebras.

$$
O \longrightarrow k \longrightarrow(\mathcal{A},\{-,-\}) \stackrel{\delta}{\longrightarrow} \mathcal{H}^{\log }(\mathcal{A}, I) \longrightarrow 0
$$

According to Dirac principle of quantization, $(\mathcal{A},\{-,-\})$ is quantization if there exist a representation $(\mathcal{H}, \varphi)$ where $\mathcal{H}$ is Lie-Rinehart extension of $\mathcal{A}$ along $\operatorname{Der}_{k}(\log I)$ such that the following diagram commute.

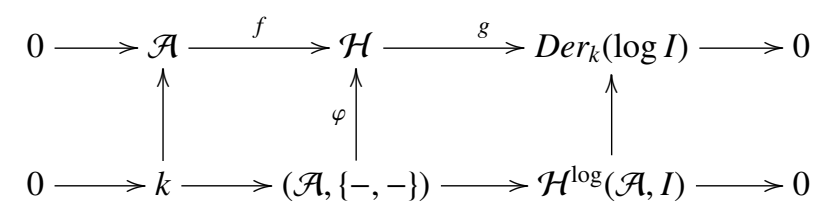

The morphism $\varphi$ is called quantization formula and it satisfies:

$$
\varphi(a s)=\nabla_{v(a)} s+2 i \pi a s
$$

$a \in \mathcal{A}, \nabla$ is a section of $g$; and $v(a)=\{a,-\}$.

In general, $\nabla$ is only an $\mathcal{A}$-module homomorphism. Obstruction to become Lie-morphism is measured by cohomology class of an 2-cocycle $K_{\nabla}$; it is usually called curvature of $\nabla$ on $\mathcal{H}$.

When $(\mathcal{H}, \varphi)$ exists and $\varphi$ satisfies (13) the triplet $\left(\mathcal{H}, \nabla, K_{\nabla}\right)$ is called prequantum representation of $\mathcal{A}$. The following paragraph is devoted to the construction of $\mathcal{H}$ when $\mu:=\omega$ is a logsymplectic structure on a even dimensional complex manifold $X$ with reduced divisor $D$.

Suppose that the logsymplectic manifold $(X, \omega, D)$ admit a prequantum representation $\left(\right.$ Diff $\left.f_{\log }^{1}(\mathcal{E}), \nabla, K_{\nabla}\right)$.

For all $f, g \in H^{0}\left(X, O_{X}\right)$ and $s \in \mathcal{E}$,

$$
\begin{aligned}
\varphi(f) \varphi(g) s & =\varphi(f)(\varphi(g) s) \\
& =\varphi(f)\left[\nabla_{v(g)} s+2 \pi i g s\right] \\
& =\nabla_{v(f)}\left(\nabla_{v(g)} s+2 \pi i g s\right)+2 \pi i\left(f \nabla_{v(g)} s+2 \pi i f g s\right) \\
& =\nabla_{v(f)} \nabla_{v(g)} s+2 \pi i \nabla_{v(f)}(g s)+2 \pi i \nabla_{v(g)} s-4 \pi^{2} f g s \\
& =\nabla_{v(f)} \nabla_{v(g)} s+2 \pi i(H(d f) . g) s+2 \pi i g \nabla_{v(f)} s+2 \pi i f \nabla_{v(g)} s-4 \pi^{2} f g
\end{aligned}
$$

Changing the role of $f$ and $g$, we obtain:

$$
\varphi(g) \varphi(f) s=\nabla_{v(g)} \nabla_{v(f)} s+2 \pi i(H(d g) . f) s+2 \pi i g \nabla_{v(g)} s+2 \pi i g \nabla_{v(f)} s-4 \pi^{2} g f s
$$

Therefore

$$
[\varphi(f), \varphi(g)] s=\left[\nabla_{v(f)}, \nabla_{v(g)}\right] s+4 \pi i \omega(v(f), v(g)) s
$$


In other hand,

$$
\begin{aligned}
\varphi(\{f, g\}) & =\nabla_{v(\{f, g\})} s+2 \pi i\{f, g\} s \\
& =\nabla_{[v(f), v(g)]} s+2 \pi i\{f, g\} s \\
& =\left[\nabla_{v(f)}, \nabla_{v(g)}\right]-K_{\nabla}(v(f), v(g)) s+2 \pi i\{f, g\} s \\
& =[\varphi(f), \varphi(g)] s+2 \pi i\{f, g\} s-K_{\nabla}(v(f), v(g)) s
\end{aligned}
$$

It follows that $\varphi$ is prequantum map of $(X, \omega, D)$ if and only if

$$
K_{\nabla}=2 \pi i \omega
$$

Since $\omega \in H^{0}\left(X, \Omega_{X}^{2}(\log D)\right)$, relation (14) implies that $K_{\nabla}$ and then $\nabla$ are logarithmic forms.

Definition 5.1. We refer to prequantum sheaf on $(X, \omega, D)$ a rank 1 connection $(\mathcal{M}, \nabla)$ satisfy (14)

\subsection{Extension of Prequantum Sheaf}

Our main objective being to determine the existence condition of prequantum sheaf $(\mathcal{M}, \nabla)$ on $(X, D, \omega)$ satisfy $(14)$, we intend in a first time to determine in which case integral condition of $\omega$ on $X-D$ could be extended to entire $X$. Of cause we shall know how and when it is possible to extend connection on $X-D$ to logarithmic connection on $D$. First about, we recall the following proposition.

Proposition 5.2. (Iitaka, S. (1981)) Let $F$ be a closed subset of a nonsingular variety $X$ with $F \neq X$ If $\omega_{1}$ and $\omega_{2}$ are rational $q$-forms such that $\left.\omega_{1}\right|_{X-F}=\left.\omega_{2}\right|_{X-F}$, then $\omega_{1}=\omega_{2}$

Proposition 5.3. If $(\tilde{\mathcal{N}}, \tilde{\nabla})$ is extension of a prequantum sheaf $(\mathcal{N}, \nabla)$ on $X^{*}=X-D$ and $D$ is a close reduced divisor of $X$, then $(\tilde{\mathcal{N}}, \tilde{\nabla})$ is a prequantum sheaf of $X$

Proof. Since $D$ is a simple normal crossing divisor and $(\tilde{\mathcal{N}}, \tilde{\nabla})$ is an extension of $(\mathcal{N}, \nabla)$ then $\left.K_{\tilde{\nabla}}\right|_{X^{*}}=K_{\nabla}=2 \pi i \omega$. The result follows from the proposition 5.2

Corollary 5.4. If $(\tilde{\mathcal{N}}, \tilde{\nabla})$ is extension of prequantum sheaf of $X^{*}=X-D$ and $D$ is simple normal crossing, then there exists strictly close logarithmic form $\tau$ such that $\tilde{\sigma}=\sigma+\tau$

Proof. From Proposition 5.3, we have; $d\left(\sigma-\sigma^{0}\right)=0$.

The existence of $\tau$ follow from lemma 4.3.

Lemma 5.5. Let $\left(\mathcal{N}, \nabla^{0}, K_{\nabla^{0}}\right)$ be a sheaf of locally free $O_{X^{*}}$ module of rank 1 . If $\left(\mathcal{M}, \nabla, K_{\nabla}\right)$ is a sheaf of locally free $O_{X}$-module of rank 1 such that

$\nabla=\nabla^{0}+\tau \otimes i d$, with $\tau$ a close logarithmic 1-form, then $\left(\mathcal{M}, \nabla, K_{\nabla}\right)$ is prequantum sheaf of $(X, \omega, D)$ if and only if

$$
K_{\nabla^{0}}=2 \pi i \omega
$$

Proof. The prequantum map (14) becomes

$$
\begin{aligned}
& \hat{:} O_{X} \rightarrow \operatorname{End}_{\mathbb{C}}(\mathcal{E}) \\
& \hat{f} s=v(f) . \tau \otimes s+\nabla^{0} s+2 \pi i f s
\end{aligned}
$$

And we have by simple calculation

$$
\begin{aligned}
\hat{f}(\hat{g} s) \quad & v(g) \tau v(f) \tau s+v(f) \tau \nabla_{v(g)}^{0} s+v(g) v(f) \tau s+2 \pi i g v(f) \tau s+v(g) \tau \nabla_{v(f)}^{0} s+ \\
& v_{v(g)} \nabla_{v(f)}^{0} s+2 i \pi g \nabla_{v(f)}^{0} s+2 i \pi v(g) \tau f s+2 i \pi f \nabla_{v(g)}^{0} s+2 i \pi v(g) f s-4 \pi^{2} g f s
\end{aligned}
$$

and

$$
\begin{aligned}
& \hat{g}(\hat{f} s) \quad=v(f) \tau v(g) \tau s+v(g) \tau \nabla_{v(f)}^{0} s+v(f) v(g) \tau s+2 \pi i f v(g) \tau s+v(f) \tau \nabla_{v(g)}^{0} s+ \\
& \nabla_{v(f)}^{0} \nabla_{v(g)}^{0} s+2 i \pi f \nabla_{v(g)}^{0} s+2 i \pi v(f) \tau g s+2 i \pi f \nabla_{v(f)}^{0} s+2 i \pi v(f) g s-4 \pi^{2} f g s
\end{aligned}
$$

then

$$
[\hat{f}, \hat{g}] s=\left[\nabla_{v(f)}^{0}, \nabla_{v(g)}^{0}\right] s+[v(f), v(g)] \tau s+4 i \pi \omega(v(f), v(g))
$$

On the other hand,

$$
\left\{\hat{f, g\}}=\left[\nabla_{v(f)}^{0}, \nabla_{v(g)}^{0}\right] s-K_{\nabla^{0}}(v(f), v(g)) s+[v(f), v(g)] \tau s+2 i \pi \omega(v(f), v(g))\right.
$$


Now, we can give the main theorem of this section.

Theorem 5.6. Let $(X, D, \omega)$ be a log symplectic manifold such that:

1. $D$ is close reduced divisor of $X$

2. $X-D$ is even dimensional complex sub manifold of $X$

3. integral of $\omega$ in all close connected surface of $X-D$ is an integer multiple of $2 \pi i$ Then the symplectic manifold $(X-D, \omega)$ is prequantizable and if its prequantum connection spreads on $X$, the $(X, D, \omega)$ is prequantizable.

Proof. Since $(X-D, \omega)$ is symplectic manifold, condition 3 of Theorem is B. Kostant quantization condition. If the prequantique sheaf of $(X-D, \omega)$ spreads on $X$, their associated curvature coincides on $X-D$. An then it follows from (5.2) that they are equal.

Since $(X-D, \omega)$ is symplectic, it is prequantizable if and only if the cohomology class $[\omega] \in H^{2}(X-D, \mathbb{C})$ live in $i_{*}\left(H^{2}(X-D, \mathbb{Z})\right)$. We shall be careful on the fact that obstruction is coming from logarithmic De Rham cohomology and not from De Rham cohomology of $X-D$. In general the two cohomologies are not equal. We need the Logarithmic Comparison Theorem before using only cohomology of $X-D$. Nowadays, it is proved that if the divisor $D$ is locally quasi-homogeneous and free, the cohomology of logarithmic De Rham complex is equal to the De Rham cohomology of the complement of divisor. If we suppose that $D$ is closed and locally quasi-homogeneous, then $X-D$ is prequantizable if the cohomology class of $\omega$ in $X-D$ is integer. We can deduce the following proposition.

Corollary 5.7. If $D$ is close, locally quasi-homogeneous and free, if the de Rham cohomology class of $\omega$ on $X-D$ is integral, then there exist a prequantum sheaf on $X-D$. Besides, if prequantum connection on $X-D$ extending on $X$, then $(X, D, \omega)$ have prequantum sheaf.

We remark that the problem of extending connection is fundamental in our approach. In the following paragraph, we will study the case where $D$ is a Normal Crossing Divisor.

\subsection{The Normal Crossing Divisor Case}

Throughout this section $X$ denotes connected complex analytic compact manifold of dimension $2 \mathrm{n}$ and $D=\sum_{i=1}^{s} v_{i} D_{i}$ an effective normal crossing divisor on X, i.e. an effective divisor locally with nonsingular components meeting transversally; $\omega$ is a logsymplectic structure on $X$. Using main result of P. Deligne and B.Malgrange about extension of connection on $X-D$ we prove the sufficient condition of prequantization of $(X, D, \omega)$.

First of all, we recall the notion of extension of connection.

Definition 5.8. If $(\mathcal{M}, \nabla)$ is connection on $X-D$, we called meromorphic extension of $\mathcal{M}$ and $O_{X}[D]$-coherent module $\tilde{\mathcal{M}}$ provided with isomorphism $\left.\tilde{\mathcal{M}}\right|_{X-D}=\mathcal{M}$

Thanks to Hilbert Nullstellensatz Theorem, B. Malgrange prove in (Malgrange, B. (1987))the following Lemma.

Lemma 5.9. (Malgrange, B. (1987)) A coherent $O_{X}[D]$-module $M$ whose support is contained in $D$ is trivial.

It follow that extension of connection $(\mathcal{M}, \nabla)$ when it exists, it is unique. The following P. Deligne Theorem assure the existence of extension of each connection on $X-D$ when $D$ is normal crossing divisor on $\mathrm{X}$.

Theorem 5.10. (Malgrange, B. (1987)) Let D be a divisor with normal crossing of $X$, and $(\mathcal{M}, \nabla)$ a connection on $X-D$. There exists a free extension $(G, \triangle)$ of $(\mathcal{M}, \nabla)$ on $X$, unique up isomorphism such that

1. $\nabla$ has logarithmic pole with respect to $G$

2. The eigenvalues of the residues of $\nabla$ with respect to $G$ belong to the image of $\tau$ the section of $\mathbb{C} \rightarrow \mathbb{C} / \mathbb{Z}$

We can then state the main theorem of this section.

Theorem 5.11. Let $(X, D, \omega)$ be a logsymplectic manifold such that:

1. D is normal crossing divisor of $X$ 
2. $X-D$ is even dimensional complex sub manifold of $X$

3. integral of $\omega$ in all close connected surface of $X-D$ is integers multiple of $2 \pi i$.

Then $(X, D, \omega)$ is prequantizable.

According to above results, if extension of each connection on $X-D$ exists, then existence of prequantum connection on $X-D$ implies that of $X$. Since the unique extension of prequantum connection $(\mathcal{M}, \nabla)$ on $X-D$ will agree on $X-D$ with $(\mathcal{M}, \nabla)$.

Lemma 5.12. If $\omega$ is exact, then $(X, \omega, D)$ is prequantizable.

Proof. If $\omega$ is exact, then $[\omega] \in H^{2}(X-D, \mathbb{C})$ is an integer. Therefore the Kostant theorem implies that there exist integrable connection $(\mathcal{N}, \nabla)$ on $X^{*}$ and from Deligne theorem, $(\mathcal{N}, \nabla)$ extend to $(\tilde{\mathcal{N}}, \tilde{\nabla})$ where the $\tilde{\nabla}$ is integrable logarithmic connection on $X$.

\section{Lie Algebroid Formalism}

Definition 6.1. (Polishchuk, A. (1997)) A Lie Algebroid on X is an $O_{X}$-module L equipped with a Lie algebra bracket [., .] and an $O_{X}$-linear morphism of Lie algebras $\rho: L \rightarrow T_{X}$ such that for $l_{1}, l_{2} \in O_{X}$ one has

$$
\left[l_{1}, f l_{2}\right]=f\left[l_{1}, l_{2}\right]+\rho\left(l_{1}\right)(f) l_{2}
$$

$\rho$ is called anchor.

We remark that Lie-Rinehart algebra is Lie-algebroid on affine scheme.

There exists many examples of Lie-algebroid in literature:

Example 6.2. $\quad 1$. Let $X$ be a smooth scheme. Then sheaf $T_{X}$ of tangent vector fields is Lie-algebroid with anchor $I d_{T_{X}}$.

2. Any real Lie-algebra $g$ is Lie-algebroid on $X=\{\bullet\}$ the associated anchor is zero map.

3. Let $(X, D)$ be a logarithmic manifold. The sheaf $T_{X}(-\log D)$ of logarithmic vector fields endowed with the inclusion morphism $T_{X}(-\log D) \hookrightarrow T_{X}$

The notion of module on Lie-Rinehart algebra is generalize as follow:

Definition 6.3. (Polishchuk, A. (1997)) Let (L, $\rho,[.,]$.$) be a Lie-algebroid. An L-module is an O_{X}$-module M equipped with Lie action; $\omega: L \rightarrow \operatorname{End}_{O_{X}}(M)$ which is a homomorphism of Lie-algebra; such that for all $f \in O_{X}, l \in L$ and $x \in M$ :

1. $(\omega(f l))(x)=f((\omega(l))(x))$

2. $(\omega(l))(f x)=\rho(l)(f) x+(\omega(f l))(x)$

To simplify the notation we will denote $(\omega(l))(x)=l(x)$

Definition 6.4. Let $(L, \rho,[.,]$.$) be a Lie-algebroid. Universal objet of L$ is a sheaf of $O_{X}$-algebras $U(L)$ equipped with a morphism $i_{O_{X}}: O_{X} \rightarrow U(L)$ of $O_{X}$-algebras and a morphism $i_{L}: L \rightarrow U(L)$ of Lie-algebras having the following properties

$$
\left[i_{L}(l), i_{O_{X}}(x)\right]=i_{O_{X}}(l(x)) \quad i_{O_{X}}(f) i_{L}(l)=i_{L}(f l)
$$

and $\left(U(L), i_{O_{X}}, i_{L}\right)$ is universal among triplet $(V, \alpha, \beta)$ satisfy 17.

The notion of universal enveloping algebra of Lie-algebroid is very useful when we define Poisson cohomology in the framework of Algebraic Geometry (Polishchuk, A. (1997)).

Another notion that we need is extension of lie-algebroid.

Definition 6.5. Let $L$ be Lie-algebroid on $X$. An extension of $L$ by and $O_{X}$-module $F$ is an exact sequence of Lie-algebroid

$$
0 \longrightarrow F \stackrel{i}{\longrightarrow} E \stackrel{\pi}{\longrightarrow} L \longrightarrow 0
$$

where $E$ is Abelian Lie-algebroid. 
Many notion are related to Lie-algebroid extension. For example:

A transverse of an extension of Lie-algebroid $L$ is a morphism of $O_{X}$-modules $\chi: L \rightarrow E$ such that $\pi \circ \chi=I d_{L}$.

A back-transverse of an extension of Lie-algebroid is a morphism of $O_{X}$-modules $\lambda: E \rightarrow L^{\prime}$ such that $\lambda \circ i=I d_{E}$

A transverse is flat if it is homomorphism of Lie-algebroid.

Proposition 6.6. There is one to one correspondence between the transverses $\chi$ and back-transverses $\lambda$ giving by

$$
i \circ \lambda+\chi \circ \pi=I d_{E} \quad \text { such that } \lambda \circ \chi=0
$$

Proof. It is an adaptation of proof in the smooth case.

When $\pi$ is a submersion of fiber bundles, a transverse all way exist and the choice of the transverse determine an isomorphism $E \backsim F \bigoplus L$. The following proposition is a generalization of Proposition 2.13 (see (Huebschmann, J. (1998, January)))

Proposition 6.7. For any Lie-algebroid $L$ and an $O_{X}$-module locally free $\mathcal{E}$, there is up to congruence extensions at most one extension of L by $\operatorname{End}_{O_{X}}(\mathcal{E})$

Proof. Let $\left(L,[-,-]_{\rho}, \rho\right)$ be an Lie algebroid on $X$ and $\mathcal{M}$ an $O_{X}$-module. Define on $\mathcal{E} n d_{\mathbb{C}}(\mathcal{M}) \oplus \mathcal{L}$ the following bracket

$$
\left[(\beta, l),\left(\beta^{\prime}, l^{\prime}\right)\right]=\left(\beta \beta^{\prime}-\beta^{\prime} \beta,\left[l, l^{\prime}\right]_{\rho}\right)
$$

For each open subset $U$ of $X$ define

$\Gamma(U, \mathcal{A}(\mathcal{M}))=\left\{(\beta, l) \in \mathcal{E} n d_{\mathbb{C}}(\mathcal{M}) \oplus \mathcal{L}, \beta(f m)=\rho(l)(f) m+f \beta(m)\right\}$.

Lemma 6.8. $\mathcal{A}$, is a sheaf of $O_{X}$-module

Indeed, for all $f \in O_{X},(\beta, l) \in \mathcal{A}(\mathcal{M})$. Define $f(\beta, l):=(f \beta, f l)$.

Since

$$
\begin{aligned}
f \beta(g m) & =f(\beta(g m)) \\
& =f(\rho(l)(g) m+g \beta(m)) \\
& =f \rho(l)(g) m+f g \beta(m) \\
& =\rho(f l)(g) m+f g \beta(m) \\
& =\rho(f l)(g) m+g((f \beta)(m))
\end{aligned}
$$

Then $f(\beta, l) \in \mathcal{A}(\mathcal{M})$.

\section{$\mathcal{A}$, is a Lie algebroid.}

Indeed, let $(\beta, l),\left(\beta^{\prime}, l^{\prime}\right) \in \mathcal{A}(\mathcal{M})$.

We have:

$$
\begin{aligned}
\beta \beta^{\prime}(f m) & =\beta(\beta(f m)) \\
& =\beta\left(\rho\left(l^{\prime}\right)(f) m+f \beta^{\prime}(m)\right) \\
& =\beta\left(\rho\left(l^{\prime}\right)(f) m\right)+\beta\left(f \beta^{\prime}(m)\right) \\
& =\beta\left(\rho\left(l^{\prime}\right)(f) m\right)+\rho(l)(f) \beta^{\prime}(m)+f\left(\beta \beta^{\prime}(m)\right) \\
& =\rho(l)\left(\rho\left(l^{\prime}\right)(f)\right) m+\rho\left(l^{\prime}\right)(f)(\beta(m))+\rho(l)(f) \beta^{\prime}(m)+f\left(\beta \beta^{\prime}(m)\right)
\end{aligned}
$$

In the same manner, we obtain

$\beta^{\prime} \beta(f m)=\rho\left(l^{\prime}\right)(\rho(l)(f)) m+\rho(l)(f)\left(\beta^{\prime}(m)\right)+\rho\left(l^{\prime}\right)(f) \beta(m)+f\left(\beta^{\prime} \beta(m)\right)$. Then $\left[\beta, \beta^{\prime}\right](f m)=\left(\rho\left[l, l^{\prime}\right] \rho(f)\right) m+f\left(\left[\beta, \beta^{\prime}\right](m)\right)$. Therefore, $\left[(\beta, l),\left(\beta^{\prime}, l^{\prime}\right)\right]=\left(\beta \beta^{\prime}-\beta^{\prime} \beta,\left[l, l^{\prime}\right]_{\rho}\right) \in \mathcal{A}(\mathcal{M})$

\section{Leibniz property}

Let $(\beta, l),\left(\beta^{\prime}, l^{\prime}\right) \in \mathcal{A}(\mathcal{M}), f \in O_{X} .\left[(\beta, l), f\left(\beta^{\prime}, l^{\prime}\right)\right]=\left(\beta f \beta^{\prime}-f \beta^{\prime} \beta,\left[l, f l^{\prime}\right]_{\rho}\right)$. We have $\beta\left(f \beta^{\prime}\right)(m)-\left(f \beta^{\prime}\right) \beta(m)=\rho(l)(f) \beta^{\prime}(m)+$ $f\left(\beta \beta^{\prime}(m)-\beta^{\prime} \beta(m)\right)$. Then $\beta\left(f \beta^{\prime}\right)-\left(f \beta^{\prime}\right) \beta=\rho(l)(f) \beta^{\prime}+f\left[\beta, \beta^{\prime}\right]$. Then $\left(\beta f \beta^{\prime}-f \beta^{\prime} \beta,\left[l, f l^{\prime}\right]_{\rho}\right)=\rho(l)(f)\left(\beta^{\prime}, l^{\prime}\right)+f()\left[\beta, \beta^{\prime}\right],\left[l, l^{\prime}\right]_{\rho}$.

Therefore the Lie Leibniz property is satisfied with anchor $\Phi(\beta, l)=\rho(l)$. 
Proposition 6.9. 1. For all $(\beta, l) \in \mathcal{A}(\mathcal{M}), \beta \in \operatorname{Diff}^{1}(\mathcal{M})$

2. If $L=\operatorname{Der}(-\log D)$ then $\beta \in \operatorname{Diff}_{\log }^{1}(\mathcal{M})$

3. the map $\varphi((\beta, l))=\beta$ is Lie-algebroid homomorphism

Proof. 1. Let $(\beta, l) \in \mathcal{A}(\mathcal{M}) . \beta(f s)=(\rho(l)(f)) s+f(\beta(s))$

Then $\beta(f s)-f(\beta(s))=(\rho(l)(f)) s$

Therefore $\beta \in \operatorname{Diff}^{1}(\mathcal{M})$

2. Suppose that $L=\operatorname{Der}(-\log D)$, then $\rho(l) \in \operatorname{Der}(-\log D)$ and then $\rho(l)(h) \in h O_{X}$ for all $l \in \operatorname{Der}(-\log D)$.

Therefore $[\beta(f s)-f(\beta(s))] h^{-1} \in O_{X}$

3. consider the map

$$
\begin{array}{rlcc}
\varphi: \quad \mathcal{A}(\mathcal{M}) & \rightarrow & \operatorname{Diff}^{1}(\mathcal{M}) \\
(\beta, l) & \mapsto & \beta
\end{array}
$$

$\varphi$ is an homomorphism of Lie-algebroid.

Indeed for all $(\beta, l) \in \mathcal{A}(\mathcal{M}), s \in \mathcal{M}$;

$$
\begin{aligned}
\sigma \circ \varphi((\beta, l))(f)(s) & =\sigma_{\varphi(\beta, l)}(f)(s) \\
& =\sigma_{\beta}(f)(s) \\
& =\beta(f s)-f \beta(s) \\
& =(\rho(l)(f))(s) \\
& =(\Phi(\beta, l)(f))(s)
\end{aligned}
$$

then $\sigma \circ \varphi=\Phi$ where $\Phi(\beta, l)=\rho(l)$

Corollary 6.10. For all $O_{X}$-module locally free of rang $1, \mathcal{M}, \operatorname{Diff}_{\log }^{1}(\mathcal{M}), \mathcal{A}_{\log }(\mathcal{M}) \in \operatorname{Ext}^{1}\left(O_{X}, \operatorname{Der}(-\log D)\right)$

Proof. It follows from the five lemma and above proposition.

It follows from this proposition that, giving a complex line bundle $L$ on a logarithmic manifold $(X, D)$, There exist up to congruence of extensions at most one extension of the Lie algebroid $T_{X}(-\log D)$

Corollary 6.11. For all $O_{X}$-module locally free of rank one $L$ there exist an exact sequence of Lie algebras

$$
0 \longrightarrow \operatorname{End}(\Gamma(\mathcal{E})) \stackrel{i}{\longrightarrow} A_{\log }(\mathcal{E}) \stackrel{\pi}{\longrightarrow} \operatorname{Der}(-\log D) \longrightarrow 0
$$

Therefore, we have the following definition

Definition 6.12. An extension $A_{\log }(\mathcal{E})$ of Der $(-\log D)$ giving by relation (21) is called Atiyah logarithmic algebroid of the invertible sheaf $\mathcal{E}$.

The existence of $A_{\log }(\mathcal{E})$ allowed us to think about representation of logsymplectic Poisson algebra $\left(O_{X}, \omega\right)$ by $A_{\log }(\mathcal{E})$. In this case prequantum representation shall commute the following diagram.

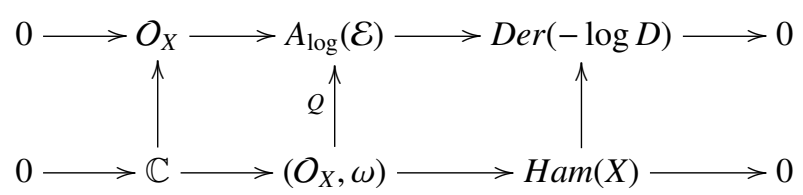

Lemma 6.13. Let $\nabla: \operatorname{Der}(\log D) \rightarrow \operatorname{End}(\mathcal{M})$ be a logarithmic connection on $\mathcal{M}$. For all $\delta \in \operatorname{Der}(-\log D)$

1. $\sigma_{\nabla_{\delta}}=\delta$

2. For all $\varphi \in \mathcal{A}_{\log }(\mathcal{M}), \nabla_{\sigma_{\varphi}}-\varphi \in \operatorname{ker}(\sigma) \bumpeq O_{X}$ 
Proof. Let $\nabla$ be a logarithmic connection on $\mathcal{M}$ and $\delta \in \operatorname{Der}(-\log D)$.

1. $\nabla_{\delta}(f s)=f \nabla_{\delta} s+\delta(f) s$; i.e. $\delta(f) s=\nabla_{\delta}(f s)-f \nabla_{\delta} s$.

Therefore, there exist $g \in O_{X}$ such that $\left[\nabla_{\delta}(h s)-h \nabla_{\delta} s\right] h^{-1}=g s$; i.e. $\nabla_{\delta} \in \mathcal{A}_{\log D}(\mathcal{M})$ we can compute its image by $\sigma$. We have:

$$
\begin{aligned}
\sigma_{\nabla_{\delta}}(f)(s) & =\nabla_{\delta}(f s)-f \nabla_{\delta} s \\
& =(\delta(f)) s \quad \forall f \in O_{X}, s \in \mathcal{M}
\end{aligned}
$$

Then $\sigma_{\nabla_{\delta}}=\delta$

2. $\forall \varphi \in \mathcal{A}_{\log }(\mathcal{M})$,

$$
\begin{aligned}
\sigma_{\nabla_{\sigma_{\varphi}}}(f)(s) & =\nabla_{\sigma_{\varphi}}(f s)-f \nabla_{\sigma_{\varphi}} s \\
& =f \nabla_{\sigma_{\varphi}} s+\left(\sigma_{\varphi}(f)\right) s-f \nabla_{\sigma_{\varphi}} s \\
& =\left(\sigma_{\varphi}(f)\right) s \\
\text { i.e., } \sigma_{\nabla_{\sigma_{\varphi}}}(f)(s) & =\sigma_{\varphi}(f) s \forall f \in O_{X}, s \in \mathcal{M} \\
\text { i.e., } \sigma_{\nabla_{\sigma_{\varphi}}}-\sigma_{\varphi} & =0 \\
\text { i.e., } \sigma_{\nabla_{\sigma_{\varphi}}-\varphi} & =0 \\
\text { i.e., } \nabla_{\sigma_{\varphi}}-\varphi & \in \operatorname{ker}(\sigma)
\end{aligned}
$$

It follows that there exist $m(\varphi) \in O_{X}$ such that

$$
\varphi=\nabla_{\sigma_{\varphi}}+m(\varphi)
$$

Lemma 6.14. Let $\omega$ be a logsymplectic form on $X$, the following morphism $\operatorname{Der}(-\log D) \rightarrow \Omega_{X}^{1}(\log D) \quad$ by $\quad \delta \mapsto i_{\delta} \omega$ is an isomorphism if $D$ is free.

It follows from this lemma that $\forall f \in O_{X}$ there exist $\delta_{f} \in \operatorname{Der}_{X}(-\log D)$ such that $i_{\delta_{f}} \omega=d f$

Lemma 6.15. There exist a map $\lambda:\left(O_{X}, \omega\right) \rightarrow \mathcal{A}_{\log }(\mathcal{M})$ who commute the following diagram

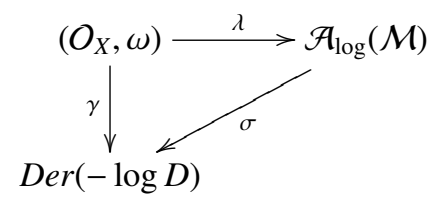

Proof. For all $f \in O_{X}$ since $\sigma$ is onto, it admit a section $\tau$ such that $\sigma \circ \tau=I d$ we denote $\lambda=\tau \circ \gamma$

For all $f \in O_{X}, \lambda_{f}$ satisfies equation (23) i.e. $\lambda_{f}=\nabla_{\sigma_{\lambda_{f}}}+m(f)$ where we have replaced $m\left(\lambda_{f}\right)$ by $m(f)$.

Corollary 6.16. $m$ is $O_{X}$-linear on $\mathcal{M}$

Proof. We have

$$
\begin{array}{lll}
\lambda_{f}(g s) & =\nabla_{\sigma_{\lambda_{f}}}(g s)+m(f)(g s) \\
& =g \nabla_{\sigma_{\lambda_{f}}} s+\sigma_{\lambda_{f}}(g) s+m(f)(g s) \\
\lambda_{f}(g s)-g \lambda_{f}(s) & =g \nabla_{\sigma_{\lambda_{f}}} s+\sigma_{\lambda_{f}}(g) s+m(f)(g s)-g \nabla_{\sigma_{\lambda_{f}}} s+g \sigma_{\lambda_{f}} \\
\lambda_{f}(g s)-g \lambda_{f}(s) & = & \sigma_{\lambda_{f}}(g)(s)+(m(f))(g s)-g(m(f))(s) \\
\text { B.e. }(m(f))(g s)-g(m(f))(s) & = &
\end{array}
$$

Corollary 6.17. $\quad$ 1. The map $Q$ in diagram $(22) Q:\left(O_{X}, \omega\right) \rightarrow \mathcal{A}_{\log }(\mathcal{M})$ have the form $Q(f)=\nabla_{\delta_{f}}+\alpha m(f)$ for some constant $\alpha$

2. $Q(f)=\nabla_{\delta_{f}}+\alpha m(f)$ is prequantization representation if and only if $m:\left(O_{X}, \omega\right) \rightarrow\left(O_{X}, \omega\right)$ is $\mathbb{C}$-linear and for any $f, g \in\left(O_{X}, \omega\right)$ and

$$
\frac{K_{\nabla}\left(\delta_{f}, \delta_{g}\right)}{\alpha}=\delta_{g} m(f)-\delta_{f} m(g)+m\{f, g\}
$$


Definition 6.18. 1. The polynomial $h\left(z_{1}, \ldots, z_{n}\right)=\sum a_{i_{1} \ldots i_{n}} z^{i_{1}} \ldots z^{i_{n}} \in O_{C^{n}}$ is weighted homogeneous if there exist positive integer weights $w_{1}, \ldots, w_{n}$ such that $h\left(z_{1}^{w_{1}}, \ldots, z_{n}^{w_{n}}\right)$ is homogeneous.

2. The divisor $D \subset X$ is locally quasi-homogeneous if for all $x \in D$ there are local coordinates on $X$, centered at $x$, with respect to which $D$ has a weighted homogeneous defining equation.

Proposition 6.19. ( Castro-Jiménez, F., Narváez-Macarro, L., E Mond, D. (1996)) Let D be a strongly quasihomogeneous free divisor in the complex manifold $X$, let $U$ be the complement of $D$ in $X$, and let $j: U \rightarrow X$ be inclusion. Then the natural morphism from the complex $\Omega_{X}^{*}(\log D)$ of differential forms with logarithmic poles along $D$ to $R j * \mathbb{C}$ is quasiisomorphism.

It follows from 6.19 and Grothendieck's Comparison Theorem that, Cohomology of $X-D$ compute the one of the complex $\left(\Omega_{X}^{*}(\log D), d\right)$. We denote $H_{d R \log }^{*}(X)$ the cohomology of the complex $\left(\Omega_{X}^{*}(\log D), d\right)$.

Let $X$ be a complex analytic space, $\mathcal{F}$ a coherent sheaf on $X$.

Denote by $\mathfrak{G}_{k}(\mathcal{F}):=\left\{m \in X ; \operatorname{prof}_{m} \mathcal{F} \leq k\right\}$.

We saying that the sheaf $\mathcal{F}$ satisfies the condition $\left(s_{k}\right)$ if

$$
\operatorname{dim} \mathfrak{G}_{k}(\mathcal{F}) \leq k-2
$$

Theorem 6.20. If $D$ is zero dimensional locally homogeneous free divisor of $X$ and if the De Rham cohomology class of $\omega$ on $X-D$ live in $i_{*}\left(H^{2}(X-D), \mathbb{Z}\right)$ then $(X, \omega)$ have prequantum bundle if the associated prequantum bundle of $X-D$ satisfies the condition $\left(s_{2}\right)$

Proof. Since the De Rham cohomology class of $\omega$ on $X-D$ live in $i_{*}\left(H^{2}(X-D)\right.$, it follow from B. Kostant in (Kostant, B. (1970)) that there exist a rank one locally free $O_{X-D}$-module $\mathcal{F}$ such that the curvature satisfies the equation 24 with $\alpha=-2 \pi i$. If $\mathcal{F}$ satisfies the condition $\left(s_{2}\right)$ and $D$ is zero dimensional analytic divisor of $X$, then according to Trautmann Theorem, there exist an unique analytic coherent sheaf $\tilde{\mathcal{F}}$ on $X$ extending $\mathcal{F}$. Since the curvature of $\mathcal{F}$ coincides on $X-D$ with curvature of $\tilde{\mathcal{F}}$ it follows from Proposition 5.2 and to the Logarithmic Comparison Theorem that $\tilde{\mathcal{F}}$ is prequantum sheaf of $X$

\section{Acknowledgments}

The author is grateful Michel Granger, Michel Nguiffo Boyom, Eugène Okassa and Metsakeu Nzoli Blondel for useful comments and discussions. He especially want to thank the Faculty of Sciences of the University of Maroua for the logistics granted at his disposal during this work.

\section{References}

Bourbaki, N. (2007). Algèbre: Chapitres 1 à 3. Springer Science \& Business Media.

Braconnier, J. (1977). Algebres de Poisson. CR Acad. Sci. Paris S ér. A Math, 284, 1345-1348.

Calderón-Moreno, F. J. (1999, September). Logarithmic differential operators and logarithmic de Rham complexes relative to a free divisor. In Annales Scientifiques de l'Ecole Normale Supérieure 32(5), 701-714. No longer published by Elsevier. https://doi.org/10.1016/S0012-9593(01)80004-5

Castro-Jiménez, F., Narváez-Macarro, L., \& Mond, D. (1996). Cohomology of the complement of a free divisor. Transactions of the American Mathematical Society, 348(8), 3037-3049. https://doi.org/10.1090/S0002-9947-96-01690-X

Chevalley, C., \& Eilenberg, S. (1948). Cohomology theory of Lie groups and Lie algebras. Transactions of the American Mathematical society, 63(1), 85-124. https://doi.org/10.1090/S0002-9947-1948-0024908-8

Deligne, P. (2006). Équations différentielles à points singuliers réguliers 163. Springer.

Dolgushev, V. (2009). The Van den Bergh duality and the modular symmetry of a Poisson variety. Selecta Mathematica, New Series, 14(2), 199-228. https://doi.org/10.1090/S0002-9947-1948-0024908-8

Dongho, J., \& Yotcha, S. R. (2016). On logarithmic Prequantization of logarithmic Poisson manifolds. Journal of Mathematical Sciences and Applications, 4(1), 4-13. https://doi.org/10.12691/jmsa-4-1-2.

Dongho, J. (2012). "Logarithmic Poisson cohomology: example of calculation and application to prequantization." Annales de la faculté des sciences de Toulouse Mathématiques 21(4), 623-650. https://doi.org/10.5802/afst.1347 
Gel'fand, I. M., \& Dorfman, I. Y. (1979). Hamiltonian operators and algebraic structures related to them. Functional Analysis and Its Applications, 13(4), 248-262. https://doi.org/10.1007/BF01078363

Goto, R. (2002). Rozansky-Witten invariants of log symplectic manifolds. Contemporary Mathematics, 309, 69-84.

Hochschild, G., Kostant, B., \& Rosenberg, A. (2009). Differential forms on regular affine algebras. Collected Papers, 265-290.

Huebschmann, J. (2013). Poisson cohomology and quantization. arXiv preprint arXiv:1303.3903.

Huebschmann, J. (1998, January). Lie-Rinehart algebras, Gerstenhaber algebras and Batalin-Vilkovisky algebras. In Annales de l'institut Fourier, 48(2), 425-440. Chartres: L'Institut.

Iitaka, S. (1981). Algebraic geometry, an introduction to birational geometry of algebraic varieties. GT M, 76.

Kostant, B. (1970). Quantization and unitary representations. Lectures in modern analysis and applications III, 87-208. https://doi.org/10.1007/BFb0079068

Krasil'shchik, I. (1988). Schouten bracket and canonical algebras. Global Analysis-Studies and Applications III, 79-110. https://doi.org/10.1007/BFb0080424

Lichnerowicz, A. (1977). Les variétés de Poisson et leurs algebres de Lie associées. Journal of différential geometry, 12(2), 253-300. https://doi.org/10.4310/jdg/1214433987

Lie, S. (1888). Theorie der transformationsgruppen (Vol. 1). (Zweiter Abschnitt, unter Mitwirkung von Prof. Dr. Friederich Engel), Teubner, Leipzig,

Lie, S. (1880). Theorie der transformationsgruppen I. Mathematische Annalen, 16(4), 441-528. https://doi.org/10.1007/BF01446218

Malgrange, B. (1987). Regular connections, after Deligne. Algebraic D-modules, 151-172.

Palais, R. (1961). The cohomology of Lie rings. In Proc. Sympos. Pure Math, 3, 130-137. https://doi.org/10.1090/pspum/003/0125867

Polishchuk, A. (1997). Algebraic geometry of Poisson brackets. Journal of Mathematical Sciences, 84(5), 1413-1444. https://doi.org/10.1007/BF02399197

Rinehart, G. S. (1963). Differential forms on general commutative algebras. Transactions of the American Mathematical Society, 108(2), 195-222. https://doi.org/10.1090/S0002-9947-1963-0154906-3

Saito, K. (1980). Theory of logarithmic differential forms and logarithmic vector fields.

Serre, J. P. (1958). Modules projectifs et espaces fibrés à fibre vectorielle. Séminaire Dubreil. Algèbre et th éorie des nombres, 11(2), 1-18.

Vaisman, I. (1991). On the geometric quantization of Poisson manifolds. Journal of mathematical physics, 32(12), 33393345. https://doi.org/10.1063/1.529446

Vinogradov, A. M., \& Krasil'shchik, I. S. (1981). What is the Hamiltonian formalism. London Math. Soc. Lecture Notes Ser, 60, 241-266.

Weinstein, A. (1983). The local structure of Poisson manifolds. Journal of differential geometry, 18(3), 523-557. https://doi.org/10.4310/jdg/1214437787

\section{Copyrights}

Copyright for this article is retained by the author(s), with first publication rights granted to the journal.

This is an open-access article distributed under the terms and conditions of the Creative Commons Attribution license (http://creativecommons.org/licenses/by/4.0/). 